\title{
Preface: 10th International Symposium on Cladocera, Lednice, Czech Republic
}

\author{
Adam Petrusek
}

Published online: 26 June 2017

(C) Springer International Publishing AG 2017

The year 2014 was special for the cladocerologist community, as scientists studying cladocerans had two opportunities to meet colleagues at specialized topical meetings focusing on this group of crustaceans, which aspired to attract a really global community. Both conferences took place within less than nine months from each other in Europe.

From 19 to 22 January 2014, the campus of the University of Birmingham hosted the EMBO Conference on the "Mighty Daphnia: Past, Present and Future“, a meeting of the Daphnia Genomics Consortium that was organised by the group of John Colbourne. This meeting had an appealing scientific programme, with nine keynote lectures (not including the hour-long opening talk by John Colbourne himself). No wonder that it attracted a substantial number of scientists involved in Daphnia genomic and evolutionary research, including numerous overseas participants from North America, Japan and China.

Guest editors: Adam Petrusek \& Piet Spaak / Proceedings of the 10th International Symposium on Cladocera

Electronic supplementary material The online version of this article (doi:10.1007/s10750-017-3263-7) contains supplementary material, which is available to authorized users.

\section{A. Petrusek ( $\square)$}

Department of Ecology, Faculty of Science, Charles

University, Prague, Czech Republic

e-mail: petrusek@natur.cuni.cz
Later that year-from 28 September to 3 October 2014 - the 10th Symposium on Cladocera was organised in the Czech Republic by the Department of Ecology, Charles University in Prague. This Symposium is a triennial international meeting that traditionally covers all topics related to the biology of cladocerans, increasingly stressing their use as model organisms. These topics include cladoceran ecology and evolutionary biology, host-parasite and predatorprey interactions, morphology, and diversity, as well as their biogeography, taxonomy and systematics. The contributions in this special volume of Hydrobiologia are a glimpse into the diversity of studies presented at the symposium.

The anniversary 10th Symposium on Cladocera returned to Central Europe where the first two meetings were held (the first in 1986 in Hungary, the second in 1989 in Slovakia), and we felt that such an event required a noble setting. Although a ballroom in a royal castle would be particularly fitting for our distinguished guests, we finally settled on a room originally occupied by noble horses-the baroque riding hall of the Lednice Chateau in south-eastern Czechia. Lednice is a small town in the heart of the Lednice-Valtice Cultural Landscape, a UNESCO World Heritage area at the border with Austria. The most well-known attraction of Lednice is the fairy tale-like chateau with an adjacent vast park styled according to old Italian and French gardens. A second noteworthy feature of the chateau complex is the 


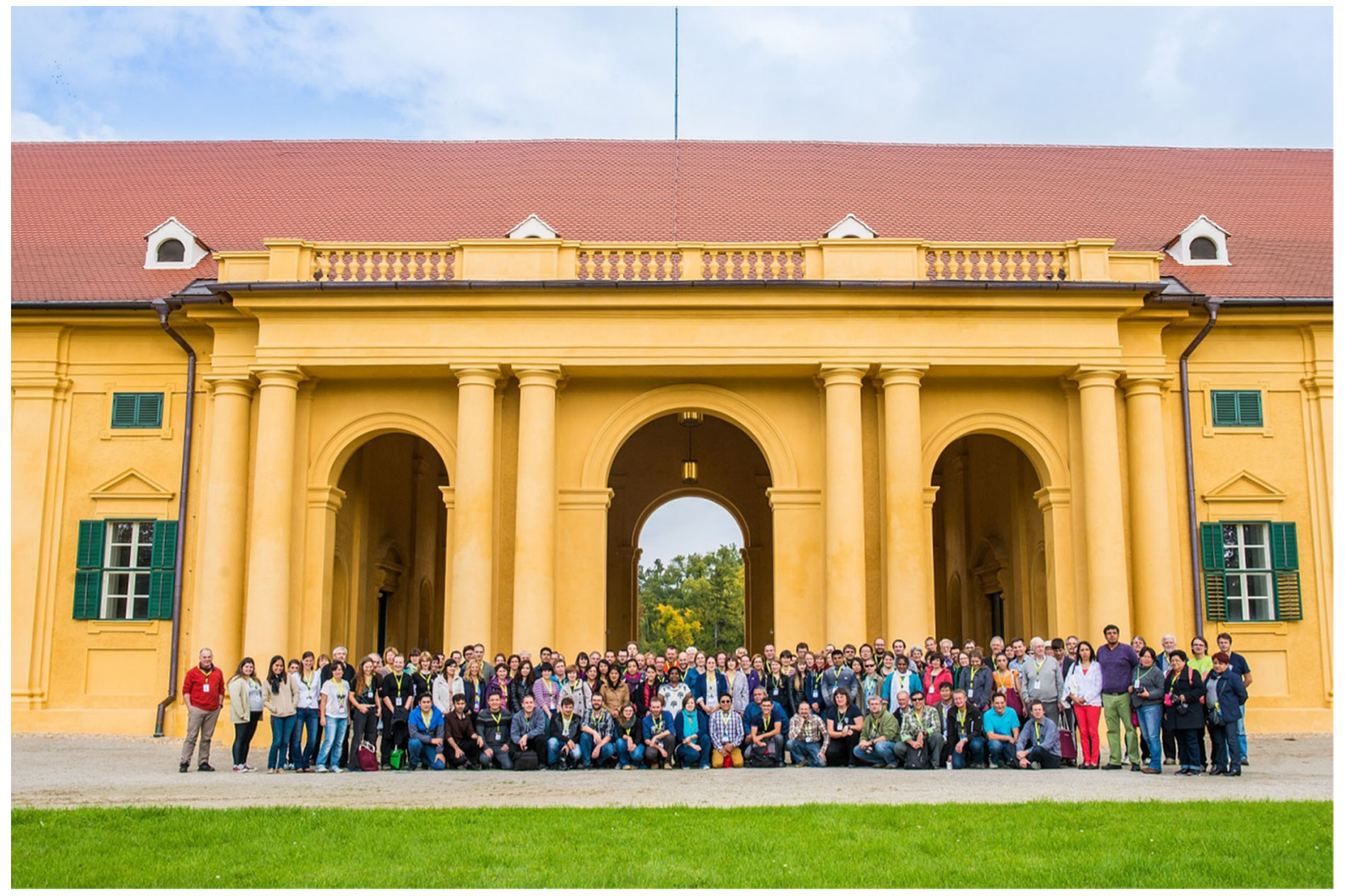

Fig. 1 Symposium participants in front of the conference venue - the historical riding hall of the Lednice Chateau. A full resolution figure is available as online supplementary information

massive baroque riding hall from the late seventeenth century, which has survived until today in an almost unaltered form. In the early 2010s it underwent a major reconstruction, during which it was converted to a multifunctional conference, information and education centre. This spectacular building (Fig. 1), which had just been opened to the public, hosted the Symposium as the very first international scientific conference held at the site. The symposium was attended by 123 participants (Fig. 1), a real global mix originating from 31 countries, including distant ones such as New Zealand, Mexico, Brazil, China, Thailand, the Philippines, Canada and the USA.

Apart from the history and culture, the LedniceValtice region and adjacent areas are also known for their vineyards and local wine. The logo of the 10th Symposium of Cladocera thus merged the tiny crustaceans that brought scientists from across the globe with the plant that attracts many other visitors to the region. A bunch of grapes with a different cladoceran genus in each berry (Fig. 2) became the symbol that accompanied symposium participants throughout the meeting. In addition to the logo, prof. Zdeněk Ďuriš, a carcinologist from the University of Ostrava, an expert on the systematics of symbiotic marine shrimps and my long-time friend, drew a set of lovely caricatures of various cladocerans that appeared in various conference materials (Figs. 3, 4).

As is the tradition of SymCla meetings, the whole symposium was organised in a single session, so the 


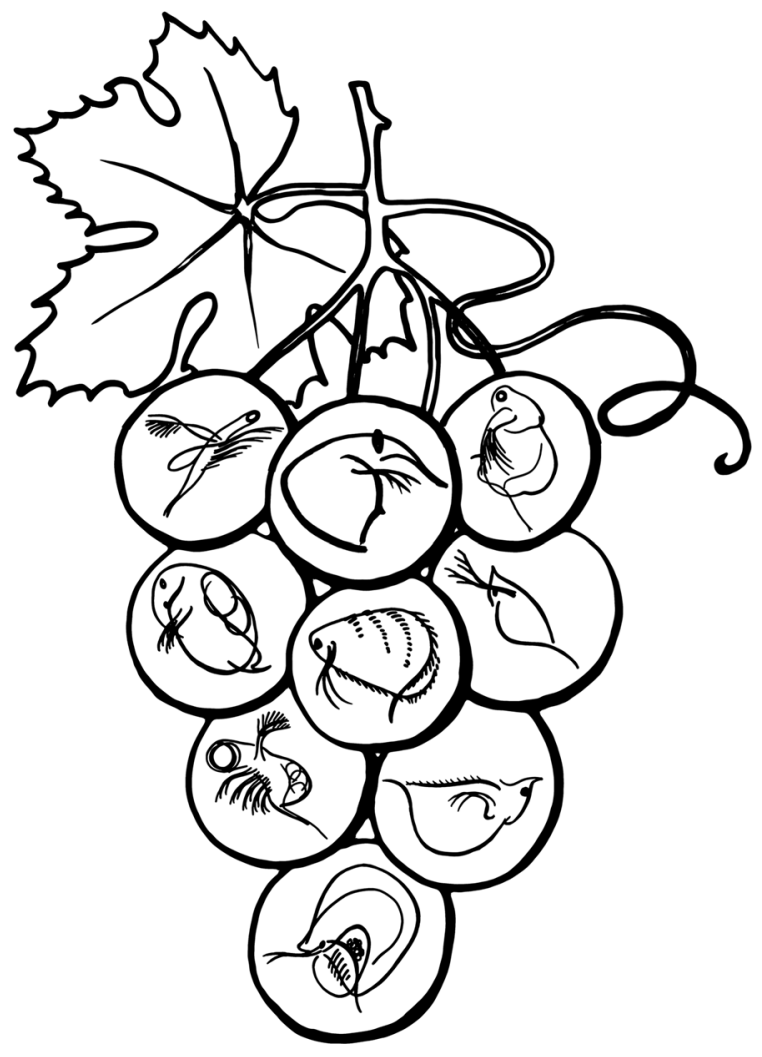

Fig. 2 Logo of the 10th Symposium of Cladocera (author: Zdeněk Duriš). Representatives of cladoceran genera, from top to bottom: Leptodora, Bosmina, Moina, Chydorus, Ilyocryptus, Daphnia, Polyphemus, Scapholeberis, Holopedium

participants could appreciate the diversity of topics in their entire breath. Kindly accepting our invitations, keynote presentations were given by two speakers, Luc De Meester (University of Leuven, Belgium) and Michael Lynch (Indiana University, Bloomington, USA). Luc and Mike gave exciting talks, on "Ecoevolutionary dynamics featuring Daphnia and cladoceran zooplankton" and "The 5000 Daphnia pulex Genomes Project", respectively. Apart from these, the programme consisted of 57 talks and almost 60 posters, as well as varied social activities, including wine tasting, folk music, a hike across limestone hills

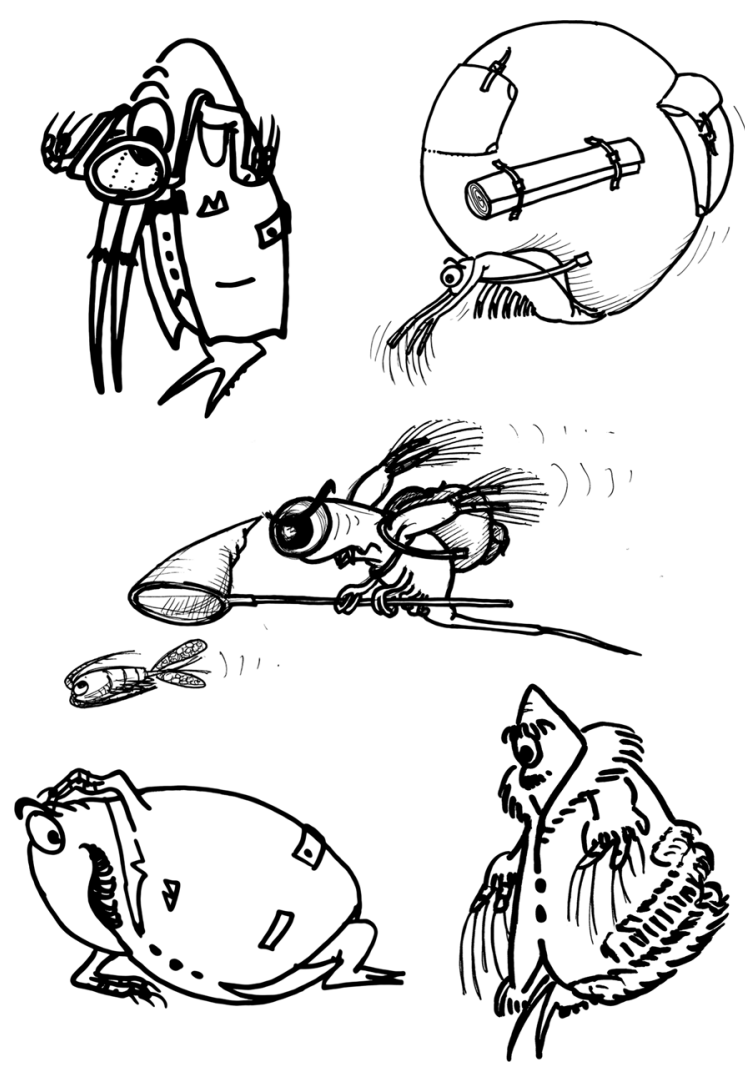

Fig. 3 Cladocerans, as you don't know them: Bosmina, Holopedium, Polyphemus, Chydorus, and Ilyocryptus (all drawings by Zdeněk Ďuriš)

or a birdwatching trip. The posters were considered to be contributions just as important as the talks. They were presented throughout the meeting in the riding hall where the talks were held, and two sufficiently long poster sessions preceded by short advertisement talks were included in the programme.

A committee composed of several senior scientists evaluated 19 student oral presentations and 21 student posters for both scientific quality and presentation skills. The following six students were awarded for outstanding contributions: Liron Goren (Tel Aviv University, Israel), Katie Sirianni (Cornell University, 


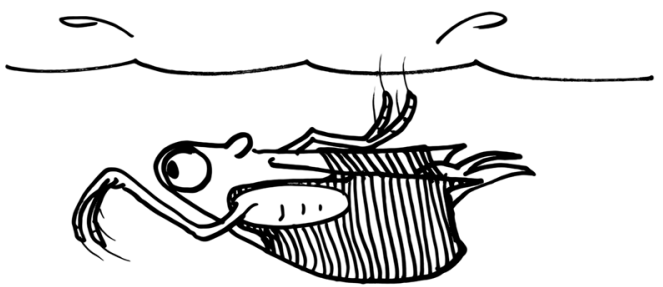

Francisco Diogo Rocha Sousa (Universidade Federal de Santa Maria, Brazil) for best posters.

At the end of the symposium, Max Rabus from University of Bayreuth volunteered to organise the 11th Symposium on Cladocera in Bavaria. Next time, the meeting will finally be held in a real castle.

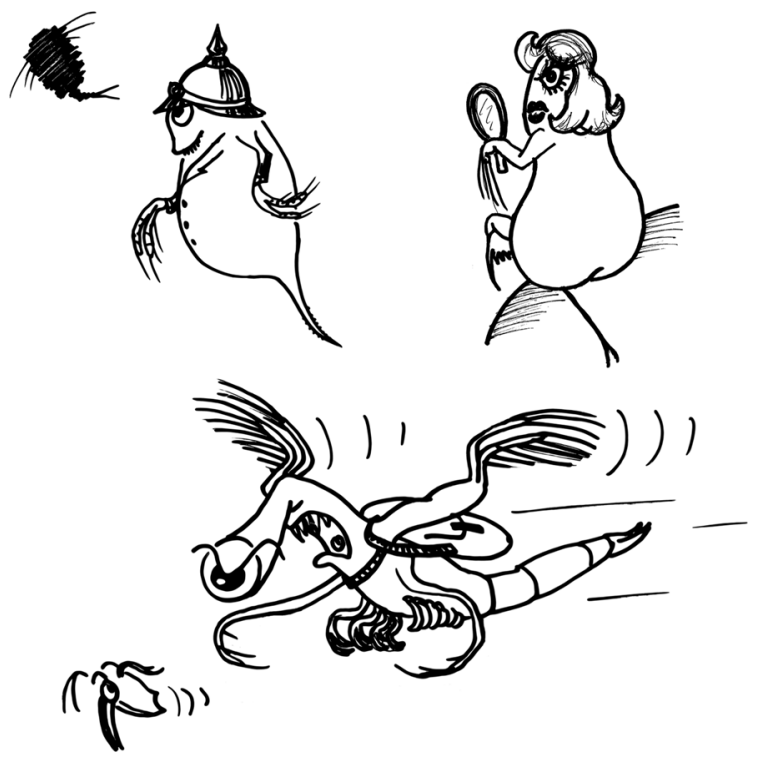

Fig. 4 Cladocerans in action: Scapholeberis, Daphnia, Moina, and Leptodora (all drawings by Zdeněk Duriš)

USA), and Sven Teurlincx (Netherlands Institute of Ecology) for best talks, and Bernard Wolfschoon Ribeiro (University of Bayreuth, Germany), Jacek Radzikowski (University of Warsaw, Poland), and 